\title{
Correction to: Frequency Based Feature Extraction Technique for Text Documents in Tamil Language
}

M. Mercy Evangeline, K. Shyamala, L. Barathi, and R. Sandhya

\author{
Correction to: \\ Chapter "Frequency Based Feature Extraction Technique \\ for Text Documents in Tamil Language" \\ in: M. Singh et al. (Eds.): Advances in Computing \\ and Data Sciences, CCIS 1441, \\ https://doi.org/10.1007/978-3-030-88244-0_8
}

In the originally published chapter 8 the name of the author was incorrect. The author's name has been corrected as "K. Shyamala". 